\title{
Avaliação das capacitações de Hanseníase: opinião de médicos e enfermeiros das equipes de saúde da família
}

\author{
Evaluation of training programs in Hansen's Disease: opinion of physicians and nurses of family health teams
}

Evaluación de las capacitaciones de Hanseníasis: la visión de médicos y enfermeros de los eQuipos de salud de la familia

\author{
Cléa Maria da Costa Moreno', Bertha Cruz Enders", Clélia Albino Simpson" \\ 'Faculdade Natalense para o Desenvolvimento do Rio Grande do Norte, Curso de Graduação em Enfermagem. Natal, RN \\ "Universidade Federal do Rio Grande do Norte. Programa de Pós-Graduação. Natal, RN
}

Submissão: 25/08/2008

Aprovação: 15/10/2008

\section{RESUMO}

A hanseníase é uma doença milenar, infectocontagiosa, causada pelo Mycobacterium leprae; manifesta-se em células cutâneas e nervos periféricos. Na década de 1990, as ações de controle foram descentralizadas, passando do estado para o município. Um estado do nordeste brasileiro elaborou, então, uma série de treinamentos em hanseníase para capacitar os profissionais da rede básica. O objetivo deste estudo foi avaliar esses treinamentos a partir da opinião de médicos e enfermeiros das equipes de saúde da família. Os resultados indicam que os profissionais avaliaram os treinamentos positivamente Quanto à sua implementação e ao objetivo de capacitá-los para a detecção da doença. Conclui-se Que os treinamentos precisam ser continuados e lançam-se algumas reflexões para os próximos.

Descritores: Hanseníase; Capacitação em serviço; Pessoal de saúde; Enfermagem.

\begin{abstract}
Hansen's Disease is a contagious, milenar disease caused by the Mycobacterium leprae that manifests itself in the cutaneal cells and the peripheral nerves. In the decade of 1990, the control for the disease was descentralized from the state to the municipality level. A northeastern state in Brazil implemented a series of training programs for health professionals in primary attention. The objective of this study was to evaluate these training programs, based on the opinions of the physicians and nurses in the family health teams. The results indicate that the professionals evaluated the training program positively as to its implementation and the achievement of its objective. It is concluded that the training programs need to continue with changes that can contribute to future programs.
\end{abstract}

Descriptors: Leprosy; Inservice training; Health personnel; Nursing.

\section{RESUMEN}

La Lepra es una enfermedad milenaria, infectocontagiosa, provocada por el Mycobacterium leprae; se manifiesta en células cutáneas y nervios periféricos. En la década del 1990, las acciones de control fueron descentralizadas, pasando de la provincia para el municipio. Una provincia del nordeste brasileño elaboró, entonces, una serie de entrenamientos en hanseniasis para capacitar a los profesionales de la red básica. El objetivo de este estudio fue evaluar esos entrenamientos, a partir de la opinión de médicos y enfermeros de los equipos de salud de la familia. Los resultados indican que los profesionales evaluaron los entrenamientos positivamente en cuanto a su implementación y el objetivo de capacitarlos para la detección de la enfermedad. Se concluye oue los entrenamientos necesitan ser continuados y se lanzan algunas reflexiones para los próximos.

Descriptores: Lepra; Capacitación en servicio; Personal de salud; Enfermería. 


\section{INTRODUÇÃO}

A hanseníase é uma doença milenar, infectocontagiosa, causada pelo Mycobacterium leprae, Que se manifesta através de sinais e sintomas dermatoneurológicos. Conhecida mundialmente como lepra, sua transmissão ocorre através das vias aéreas superiores.

A lepra é mencionada em um dos tratados médicos chineses mais antigos, o Nei Ching Su Wen, atribuído ao imperador Huang Ti, Que viveu entre 2698 e 2598 a.C.('). Está citada nos textos bíblicos, como algo a ser temido, devendo os portadores ser isolados para evitar o contágio, pois, naQuela época, não se conhecia outra forma de controle.

Por tratar-se de uma patologia Que, por muitos anos, permaneceu incurável, trouxe consigo essa carga incomensurável de estigma, Que até hoje persiste ${ }^{(2)}$. Como nos tempos bíblicos, ainda na segunda metade do Séc. XX, os pacientes de hanseníase eram isolados do convívio em sociedade, nos chamados leprosários ${ }^{(3)}$.

De acordo com a Organização Mundial de Saúde (OMS), nas décadas de 1980 e 1990, a carga global de casos de hanseníase diminuiu em Quase $90 \%{ }^{(4)}$. Apesar disso, a hanseníase ainda é considerada um grave problema de saúde pública por seu potencial incapacitante e por ser uma doença contagiosa ${ }^{(2)}$.

Em 1991, durante a 44a Assembléia Mundial de Saúde, estabeleceu-se como estratégia a eliminação da hanseníase como problema de saúde pública até o final do ano 2000 , definindo como indicador de eliminação um coeficiente de prevalência de menos de um caso por 10.000 habitantes $^{(5)}$. Essa meta não foi atingida em nível global e foi postergada para o ano de 2005, mas atualmente essa meta de eliminação foi abandonada, sendo orientadas as estratégias de controle da doença. Assim, a detecção e o tratamento precoces são considerados elementos básicos para o seu controle ${ }^{(5)}$.

No Brasil, a doença faz parte das prioridades de gestão do Ministério da Saúde (MS), através do Programa de Controle da Hanseníase (PCH).

Nos estados brasileiros, na década de 1990, com a efetivação da descentralização, um dos requisitos básicos constantes da Norma Operacional Básica de 1996 (NOB/96), o (PCH), foi repassado para a responsabilidade dos municípios ${ }^{(6)}$. A Portaria Ministerial $N^{\circ}$ 1073/ GM de 26 de setembro de 2000 orienta sobre a implantação do Programa de Controle da Hanseníase na atenção básica ${ }^{(7)}$. Para Que esses municípios assumissem, de fato, as ações de controle da doença, era necessário capacitar os profissionais da rede básica de saúde.

Para isso, o PCH do Estado do Rio Grande do Norte (RN) elaborou um projeto de treinamentos, designado como Treinamentos de Clínica em Hanseníase, Que iniciou em ainda em 1997. O objetivo principal era capacitar os profissionais de saúde para Que assumissem as ações de controle da hanseníase em nível municipal. Dessa forma, os treinamentos deveriam aumentar o conhecimento dos profissionais sobre a patologia, para eue fossem capazes de diagnosticar, tratar, prevenir incapacidades físicas e, principalmente, evitar o surgimento das formas mais graves da doença ${ }^{(2)}$.

A iniciativa de investir nas capacitações para o controle da doença foi baseada no conceito de educação permanente em saúde, Que guarda semelhança com a educação de jovens e adultos defendida pelo educador Paulo Freire. Trata-se da noção de aprendizagem significativa como base da educação permanente ${ }^{(8)}$.

Foram iniciados, assim, os treinamentos de clínica em hanseníase para os profissionais médicos e enfermeiros das unidades básicas de saúde dos municípios pertencentes às áreas endêmicas da doença. Posteriormente esses treinamentos foram direcionados para médicos e enfermeiros das Equipes de Saúde da Família (ESFs). No entanto, desde essa época, nenhuma avaliação foi realizada.

O enfoque avaliativo é importante, tendo em vista a realidade observada Quanto à situação favorável do Estado com relação à prevalência, pois, de acordo com a Carta de Eliminação do Estado, no Rio Grande do Norte, a eliminação da doença já foi alcançada ${ }^{(9)}$. Adicionalmente, segundo dados da Secretaria Estadual de Saúde, tem-se observado o aumento da detecção de pacientes com hanseníase após o início dos treinamentos realizados em 1997 até hoje, em 2007. Acredita-se Que tal aumento pode estar associado ao aperfeiçoamento dos profissionais no diagnóstico da doença. A avaliação dos treinamentos de hanseníase realizados para esse propósito traria conhecimento sobre a efetividade dessa programação educacional. Com base na importância epidemiológica da hanseníase no Estado e na necessidade de manter o nível controlável na póseliminação, considera-se Que as ações educativas bem sucedidas precisam ser mantidas.

Embora muito se tenha avançado em relação ao tratamento, principalmente após o advento da poliQuimioterapia, a manutenção do treinamento de recursos humanos é pertinente, especialmente nos aspectos de diagnóstico. Ainda é comum se ouvirem relatos de pacientes Que enumeram a Quantidade de profissionais da área de saúde pelos Quais passaram sem Que a doença fosse descoberta. A maioria relata os vários tipos de tratamentos Que realizaram, sem obterem nenhum resultado. Existem depoimentos de profissionais Que referem não se sentirem capazes de acompanhar pacientes com hanseníase, devido ao conhecimento insuficiente Que têm sobre a doença.

O estudo aQui apresentado faz parte de uma avaliação maior dos treinamentos realizados pelo PCH no Estado do Rio Grande do Norte. O estudo de base avalia o objetivo de capacitação na detecção, identificando o número de casos detectados no período antes, durante e depois dos treinamentos e a visão dos profissionais acerca dos treinamentos realizados.

A avaliação em serviços de saúde foi instituída pelo MS, a partir de 2004 com a criação do Programa Nacional de Avaliação de Serviços de Saúde (PNASS), visando possibilitar ao gestor municipal o uso de uma ferramenta de avaliação Que pudesse modificar pontos considerados críticos a partir da visão dos profissionais e usuários desses serviços ${ }^{(10)}$. A percepção do usuário já foi avaliada em outra peseuisa avaliativa, realizada em 2006, no Estado do Rio Grande do Norte ${ }^{(1)}$. Neste artigo, focaliza-se a avaliação da implementação dos treinamentos, do ponto de vista dos médicos e enfermeiros capacitados.

Assim, o Questionamento norteador deste estudo foi o seguinte: Qual a avaliação Que os profissionais médicos e enfermeiros das ESFs treinados pelo $\mathrm{PCH}$ estadual fazem acerca desses treinamentos e de suas próprias atuações nas ações de controle da hanseníase? O objetivo foi identificar a opinião dos profissionais Que participaram dos treinamentos de clínica em hanseníase no Estado do Rio Grande do Norte, acerca do curso realizado e das suas habilidades de detecção da doença como resultado do curso.

Com base nos dados da Secretaria do Estado da Saúde Pública (SESAP) e na experiência pessoal, como enfermeira assistencial e educadora, esta pesQuisa é considerada de relevância científica e 
social, principalmente, porQue se espera prestar uma contribuição para as inúmeras pessoas Que ainda permanecem sem diagnóstico preciso nem tratamento adequado. Por outro lado, observa-se que há escassez de estudos Que visem à avaliação das capacitações realizadas com os profissionais das ESFs.

Entende-se Que os principais atributos para a avaliação em saúde são: efetividade, impacto, eficácia, Qualidade, eficiência, acessibilidade, equidade, cobertura e satisfação do usuário ${ }^{(12)}$. O Ministério da Saúde elaborou um manual sobre avaliação em Que refere ao conceito de Contrandriopoulos Que considera a avaliação como um julgamento Que se faz sobre uma intervenção ${ }^{(10)}$.

Nesse sentido, o MS alinha-se, no plano internacional, ao movimento pela institucionalização da avaliação Que vem sendo implementado em diversos países, tais como Estados Unidos, Inglaterra, Canadá e França ${ }^{(10)}$. Escolheu-se, portanto, a análise da intervenção para esta pesQuisa, Que é definida como: "um estudo da relação existente entre os objetivos da intervenção e os meios empregados para alcançá-los”(13).

\section{METODOLOGIA}

Trata-se de um estudo Quantitativo. É uma investigação Que utiliza a Quantificação na forma de coletar os dados e técnicas de estatística, tanto descritivas como inferenciais, para o tratamento dos mesmos ${ }^{(14)}$.

A pesquisa foi realizada nas secretarias estadual e municipais de saúde dos municípios-sede das Unidades Regionais de Saúde Pública (URSAPs) do Estado do Rio Grande do Norte. Seis URSAPs estão localizadas no interior e uma no município de Natal, capital do Estado. Os municípios foram selecionados por terem sido locais de treinamento de clínica em hanseníase e por se entender que suas posições geográficas permitiriam uma grande abrangência territorial no Estado. Além disso, esses municípios possuem 10 ou mais ESFs, concentrando um número significativo de profissionais, o Que facilita a acessibilidade.

Os profissionais, médicos e enfermeiros que atuavam nas ESFs dos sete municípios selecionados constituíram a população do estudo. Considerando o número de equipes em cada município, Que totaliza 222, estimou-se Que essa população seria em torno de 444 profissionais. Utilizando parâmetros estatísticos para populações finitas, calculou-se, então, o tamanho da amostra de profissionais em 101 .

A representação de cada município foi baseada na proporção em Que ele contribuía para a população de equipes. Essa mesma proporção foi utilizada para determinar o número de profissionais entrevistados em cada município. A seleção do número de profissionais em cada município se deu de forma intencional, ou seja, escolheram-se apenas os Que foram treinados em hanseníase pelo PCH estadual. Todos os treinados Que se encontravam no local eram entrevistados. Atingido o número de profissionais determinado para o município, cessava-se a busca naquele local.

Foram estudadas variáveis demográficas e de trabalho, bem como as opiniões dos profissionais sobre o treinamento de clínica em hanseníase ministrado pelo PCH-RN. O instrumento utilizado foi a entrevista semi-estruturada com o auxílio de um roteiro de Questões sobre o curso e sobre suas ações de detecção.

Os dados foram coletados no período de julho de 2007 a abril de 2008, após receber parecer favorável do Comitê de Ética da
Universidade Federal do Rio Grande do Norte, através do protocolo de número 116/06. Todos os profissionais assinaram o termo de consentimento livre e esclarecido. A análise Quantitativa descritiva foi realizada através do programa EPI INFO, versão 3.4.3. Este estudo é parte de uma dissertação e obteve financiamento do CNPQ; uma das orientações será a publicação dos resultados.

\section{RESULTADOS E DISCUSSÃO}

\section{Os Profissionais e as URSAPs de Trabalho}

Foram entrevistados 98 pessoas, sendo 27 médicos e 7l enfermeiros. Isto constituiu $91,5 \%$ do total de 107 profissionais treinados, encontrados nas sete URSAPs sob estudo.

Observou-se que o número de profissionais treinados, encontrados nos municípios de cinco URSAPs interioranas, não foi o esperado, pois, de acordo com os dados da Secretaria Estadual de Saúde, 100\% dos profissionais desses municípios já tinham recebido treinamento para hanseníase. Nas URSAPs Que caracterizam a capital do Estado e a do segundo maior município, Mossoró, observou-se um maior número de profissionais com vínculo empregatício efetivo, fator Que contribuiu para sua permanência nesses municípios por mais tempo.

O fato de não encontrar todos os profissionais treinados nos municípios da amostra pode estar relacionado, sobretudo, à grande rotatividade dos profissionais das ESFs que, muitas vezes, dependem da decisão política dos gestores na designação e permanência nos locais de trabalho. Alguns procuram locais Que oferecem melhores condições de trabalho e melhores salários. Essa rotatividade foi constatada Quando se Questionou sobre o local de trabalho do primeiro treinamento de hanseníase. Observou-se que 15,3\% realizaram o treinamento em outros municípios.

Quando Questionados acerca da duração do treinamento em clínica de hanseníase, os profissionais referiram-se a cursos com duração de, em média, 27,6 horas, variando de 8 a 80 a carga horária. Alguns desses cursos foram realizados há 20 anos. Essa falta de padronização de carga horária e o tempo de realização levantam a suspeita de Que os treinamentos, realizados há mais de 10 anos, não eram de clínica em hanseníase, tendo em vista Que os treinamentos oferecidos pelo PCH-RN iniciaram em 1997 e possuíam carga horária de 16 horas.

Dentre os entrevistados, 90,8\% responderam Que atuam nas ações de controle, mas 9,2\% responderam Que não, o Que causou surpresa, pois é preconizada pelo Ministério da Saúde a inclusão do PCH nas ações de atenção básica. Essa fragilidade de controle da hanseníase na atenção básica também foi constatada no Estado de Mato Grosso, onde 50\% das ESFs não realizavam ações de diagnóstico e controle da doença ${ }^{(15)}$.

Entende-se que a Estratégia de Saúde da Família faz parte da atenção básica e Que as equipes atuam diretamente em ações de promoção da saúde, prevenção, recuperação, reabilitação de doenças e agravos mais freqüentes, bem como na manutenção da saúde da comunidade à Qual assiste ${ }^{(15)}$. A ausência dessas ações contribui para a demora no diagnóstico e a disseminação da doença, além de elevar o número de incapacidades físicas, Que podem ser irreversíveis no diagnóstico tardio ${ }^{(15)}$.

\section{Avaliação da Assistência ao Portador de Hanseníase na ESF \\ A avaliação dos profissionais entrevistados sobre a assistência}


prestada pela ESF-RN aos pacientes de hanseníase foi positiva, 59,2\% consideram-na boa e $11,2 \%$, ótima.

Dentre os Que consideraram boa a própria assistência, a maioria respondeu Que estava atendendo ao portador de hanseníase conforme os padrões esperados pelo $\mathrm{PCH}$. Os Que responderam Que a consideravam ótima referiram, principalmente, Que o acesso era adęuado, a referência e contra-referência funcionavam e a equipe trabalha ao lado do Agente Comunitário de Saúde (ACS). Os profissionais Que a consideraram ruim, péssima ou boa atribuíram isso ao fato de não haver casos na área de abrangência da sua equipe e à falta de apoio da Secretaria Municipal de Saúde.

\section{Avaliação da Atuação de Médicos e Enfermeiros das ESFs-RN nas Ações do PCH}

Foi Questionado como consideravam a atuação dos médicos e enfermeiros das ESFs do Rio Grande do Norte nas ações de controle da hanseníase. Procurava-se, dessa forma, observar Qual a percepção Que eles tinham uns dos outros.

A maioria oscilou entre boa e regular. Os Que responderam boa relataram, em sua maioria, Que consideram os profissionais bem atuantes e Que fazem diagnóstico rápido, seguido da resposta; Que os enfermeiros atuam bem, mas os médicos têm preconceito e precisam se Qualificar mais. Sobre a resposta regular, os profissionais responderam Que seria por falta de compromisso, principalmente dos médicos; há deficiência na realização de campanhas educativas, grande rotatividade de profissionais e dependência dos hospitais de referência.

Solicitou-se ainda Que fossem feitas sugestões para melhorar as ações desses profissionais. As respostas Que apareceram com maior fręüência foram: aumentar a freeüência de treinamentos, realização de campanhas educativas, sensibilização e mais compromisso dos médicos e enfermeiros.

\section{Avaliação da Capacidade dos Profissionais no Desenvolvimento das Ações de Controle da \\ Hanseníase, Antes e Depois do Treinamento}

A maioria respondeu Que não se sentia capacitada para desenvolver as ações de controle dessa doença antes de receber o treinamento de clínica em hanseníase, porQue não havia se aprofundado na patologia; tinha muitas dúvidas; medo da doença; não via pacientes na rotina; achava complicado e, na faculdade, as aulas haviam sido muito superficiais.

Sobre a capacidade de desenvolver as ações de controle depois do treinamento, 90,8\% responderam Que sim. Os motivos relacionados foram, em sua maioria, Que o treinamento serviu para reciclar e tirar dúvidas. Outros responderam Que, mesmo após o treinamento, ainda se sentem inseguros. Esses dados demonstram a carência de treinamentos, pois os profissionais da rede ainda sentem dificuldade nas ações de controle da hanseníase. A educação permanente é uma necessidade na prática cotidiana do profissional de saúde ${ }^{(8)}$.

Solicitou-se a avaliação dos profissionais médicos e enfermeiros em suspeitar de hanseníase em pacientes antes e depois de receberem o treinamento de clínica em hanseníase. Na tabela I são apresentados os resultados.

Quando Questionados sobre se, dessas suspeitas, alguma havia sido confirmada, 72,5\% responderam Que sim. A suspeição diagnóstica da hanseníase contribui muito para a resolução de muitos casos Que permanecem sem diagnóstico. Há uma expressão conhecida
Tabela 1. Distribuição dos profissionais médicos e enfermeiros das ESFs entrevistados, Quanto ao seu sentimento de capacidade para suspeição diagnóstica de hanseníase, antes e depois de treinados pelo PCH-RN. Rio Grande do Norte, 2008.

\begin{tabular}{llll}
\hline Suspeição diagnóstica & Sim & Não & Total \\
\hline Antes & 53,1 & 46,9 & 100,0 \\
Depois & 84,7 & 15,3 & 100,0 \\
\hline
\end{tabular}

pelos hansenólogos como “iceberg epidemiológico", pelo Qual se designa o fato de que o número de casos conhecidos da doença é sempre apenas a extremidade visível de contingente muito mais numeroso ${ }^{(15)}$.

\section{Avaliação dos Treinamentos EnQuanto Estratégia do PCH-RN}

Os profissionais foram Questionados ainda como avaliavam a estratégia do PCH-RN para a formação e educação permanente em relação às ações de controle da hanseníase.

A maioria dos entrevistados faz uma avaliação positiva sobre a estratégia de educação permanente do PCH-RN. Quando solicitados a justificar a resposta, a maioria respondeu Que os palestrantes eram comprometidos, sensibilizavam os participantes do treinamento para o problema da hanseníase e o material usado no curso era bom. lá os Que consideraram regular e ruim, responderam que os treinamentos no final do ano atrapalham a participação dos profissionais; Que os treinamentos são pouco freeüentes e há poucas vagas.

Perguntados também sobre a capacidade das equipes de treinamento do PCH-RN, 99\% responderam Que a equipe da Qual receberam o treinamento estava bem capacitada e apenas $1 \%$ considerou Que não.

Perguntados se a carga horária de 16 horas/aula para o treinamento de clínica em hanseníase era suficiente, 24,7\% responderam Que sim e 75,3\%, não. Solicitou-se, então, sugestão de horas/aula para o treinamento de hanseníase.

As respostas variaram de 20 a 120 horas, sendo Que a sugestão mais freeüente foi 40 horas, seguido, de 24 horas/aula. Observouse ainda Que a maioria dos médicos prefere manter a carga horária atual de 16 horas/aula dos treinamentos de clínica em hanseníase do PCH-RN.

A metodologia dos treinamentos foi avaliada de maneira positiva e, Quando solicitados a justificar, as respostas variaram como: clareza, objetividade, didática adeeuada, bons recursos audiovisuais e nas sugestões; a maioria colocou a necessidade de mais prática com pacientes.

Quanto ao conteúdo dos treinamentos, este também foi avaliado positivamente. Os entrevistados responderam Que os temas são relevantes, contemplam toda a informação necessária da clínica à prevenção de incapacidades e o material educativo é adequado. A maioria sugeriu o aumento da carga horária, pelo fato de ser uma patologia de conteúdo muito extenso.

\section{Avaliação da Contribuição dos Treinamentos para o Aumento da Detecção da Doença e as Sugestões de Mudança \\ Sobre a avaliação da contribuição dos treinamentos para o aumento}


da detecção, 97 dos 98 entrevistados responderam Que o treinamento contribui para o aumento da detecção da hanseníase. Justificaram a resposta, afirmando Que o treinamento sensibiliza os profissionais, estimula um "novo olhar" sobre a doença, responsabiliza sobre a situação epidemiológica; Que sempre Que se faz campanha de busca ativa, aparecem casos novos e enfocam-se mais os assuntos profissionais.

As sugestões oferecidas pelos profissionais entrevistados Que apareceram com maior freeüência, acerca dos treinamentos do PCHRN, foram: aumentar carga horária e freqüência dos treinamentos; treinar toda a ESF; envolver gestores e outras lideranças; sensibilizar médicos; realizar campanhas educativas e estimular referência e contra-referência

\section{CONCLUSÃO}

Neste estudo, os profissionais Que cursaram os treinamentos de clínica em hanseníase, em geral, avaliaram-nos positivamente Quanto à sua implementação objetivo principal de capacitar os enfermeiros e médicos das ESFs de sete municípios do Rio Grande do Norte na detecção da doença. No entanto, um grupo permanece com insegurança para o diagnóstico e indica a necessidade de continuação dos treinamentos. Consideram a sua atuação e a de seus colegas no controle da hanseníase adecuada, salientando, porém, a necessidade de maior envolvimento da categoria médica no processo de detecção. Avaliam a Qualidade dos treinamentos ministrados pelo $\mathrm{PCH}-\mathrm{RN}$ como boa e competente, mas consideram insuficiente a carga horária de 16 horas.

Com base nos dados coletados e na experiência profissional, foi possível avaliar o treinamento de clínica em hanseníase realizado pelo PCH-RN do ponto de vista daqueles que o vivenciaram. A pesquisa realizada sugere mudanças Que possam contribuir positivamente para os treinamentos de clínica em hanseníase do PCH-RN. Uma dessas sugestões é o aumento da carga horária dos treinamentos para 24 horas, ( 12 horas direcionadas à teoria e 12 à prática), pois, apesar de a maioria dos entrevistados ter sugerido 40 horas/aula, deve-se reconhecer Que realmente há dificuldade em deslocar as equipes por muito tempo das suas unidades.

Conclui-se Que há necessidade da continuação da educação permanente junto aos profissionais da atenção básica, com enfoque em hanseníase, considerando a sua importância para a saúde pública brasileira. A capacitação profissional demanda bons treinamentos e a conscientização Que se pode alcançar através da educação permanente. Esta reflexão, realizada no próprio serviço, estimula muito mais o profissional. Tudo leva a crer Que não há um caminho mais seguro a percorrer a não ser o da educação em saúde.

São muitas as dúvidas Que rodeiam a todos em relação a essa doença tão antiga. Mas, atualmente, há uma certeza: só depende de cada um assumir o compromisso com a prática diária, pois hanseníase tem cura. Com essa percepção e o resultado das avaliações deste trabalho, espera-se ter contribuído para o desenvolvimento das ações de controle da hanseníase.

\section{REFERÊNCIAS}

1. Opromolla DVA. Noções de hansenologia. Bauru: Centro de Estudos Dr. Reynaldo Quagliato; 2000.

2. Ministério da Saúde (BR). Secretaria de Políticas de Saúde, Departamento de Atenção Básica. Guia para o controle da hanseníase. Brasília: Ministério da Saúde; 2002.

3. Nobre ML. Projeto de pesquisa dos indicadores operacionais e epidemiológicos das ações de controle da hanseníase no Estado do Rio Grande do Norte. Natal: UNESCO; 2004.

4. World Health Assembly. Leprosy Resolution WHA 44.9. Fortyfourth World Health Assembly, 13 May 1991. Geneva: World Health Organization; 1991.

5. Organização Mundial de Saúde. Estratégia global para aliviar a carga da hanseníase e manter as atividades de controle da hanseníase. Plano 2006-20 I 0. Geneva: Organização Mundial de Saúde; 2005

6. Ministério da Saúde (BR). Saúde no Brasil: norma operacional básica/96. Brasília: Ministério da Saúde; 1997.

7. Ministério da Saúde (BR). Coordenadoria Nacional para Integração da Pessoa Portadora de Deficiência (CORDE). Brasília: Ministério da Saúde; 2000. [citado 6 out 2008]. Disponível em: http://www.mj.gov.br/sedh/ct/CORDE/dpdh/sicorde/ port_ms_1073.asp.

8. Ministério da Saúde (BR). Secretaria de Vigilância em Saúde. Plano Nacional de Eliminação da Hanseníase. IV Carta da eliminação da hanseníase - Rio Grande do Norte; 2005. [citado em 30 abr 2007]. Disponível em: http://portal.saude.gov.br/ portal/arQuivos/pdf/ riograndedonorte4.pdf

9. Ministério da Saúde (BR). Programa Nacional de Avaliação de Serviços de Saúde. PNASS. Brasília; 2004. [citado 24 abr 2008]. Disponível em: http://pnass.datasus.gov.br/documentos/ CADERNO_PNASS.pdf.

10. Simpson CA, Moreno CMC, Alencar L, Arboés AA. Fatores Que interferem nas ações do programa da hanseníase em municípios do Estado do Rio Grande do Norte. Natal: Leprosy Relief Association; 2006.

11. Silva LMV, Formigli VLA. Avaliação em saúde: limites e perspectivas. Cad Saúde Pública 1994; 10(1): 80-9.

12. Hartz ZMA. Avaliação em saúde: dos modelos conceituais à prática na análise da implantação de programas. Rio de Janeiro: Fiocruz; 2000.

13. Richardson RJ. Pesquisa social: métodos e técnicas. São Paulo: Atlas; 1999.

14. Canesqui AM, Spinelli MAS. Saúde da família no Estado de Mato Grosso, Brasil: perfis e julgamentos dos médicos e enfermeiros. Cad Saúde Pública 2006; 22(9): 1 88 1 - 1892.

15. Opromolla DV, Nóbrega RC, Gonçalves NNS, Padovani SHP, Padovani CR, Gonçalves A. Estimativa da prevalência da hanseníase pela investigação em demanda inespecífica de agências de saúde. Rev Saúde Pública 1990; 24(3): 178-85. 